\title{
Relación entre el rendimiento académico y autoconcepto en jugadoras de baloncesto de categoría cadete en competición nacional extraescolar
}

\section{Relationship between the academic achievement and the self-concept in young basketball players in extracurricular national competition}

\author{
Fátima Chacón Borrego ${ }^{1}$, Rosario Padial Ruz², Sergio Yedra Clements 3 , Sara Bretón Prats 4 , \\ Mar Cepero González y Félix Zurita Ortega ${ }^{2}$ \\ 1 Departamento de Educación Física y deporte. Universidad de Sevilla. \\ 2 Departamento de Didáctica de la Expresión Musical, Plástica y Corporal. Universidad de Granada \\ 3 Graduado en Primaria. Universidad de Granada \\ 4 Investigadora Grupo HUM-727
}

\begin{abstract}
Resumen: Esta investigación tiene como objetivo estudiar las relaciones existentes entre la actividad física, el autoconcepto y el rendimiento académico enjugadoras de baloncesto en categoría cadete. Es un estudio de tipo descriptivo y de corte transversal, realizado sobre una muestra de 75 escolares con edades de entre 12 y 16 ańos. Se han empleado como instrumentos de análisis los cuestionarios: AF-5de García y Musitu (1999) yuna hoja de autoregistro (en el que se incluyen las calificaciones académicas y tiempo de práctica de actividad física). Los resultados revelan que la práctica deportiva favorece el autoconcepto académico, así como el rendimiento académico. La dimensión autoconcepto familiar se ve claramente afectada si se dedica mucho tiempo a la realización de actividad física. Aunque el número de horas de entrenamiento no influyen directamente en el rendimiento académico, sí se puede concluir que la práctica de actividad física mejora principalmente el autoconcepto y el rendimiento académico.

Palabras clave: autoconcepto; rendimiento académico; actividad física;baloncesto; escolares.
\end{abstract}

Abstract: This research aims to study the relationship between the physical activity, self-concept and academic achievement ofparticipant's basketball players in a women's championship national category. This descriptive and cross-sectional study was conducted on a sample of 75 students between 12 and 16 years old of subject using as main tools the questionnaire: AF-5, sociodemographic(it includes academic qualifications and physical activity). On one hand, the result showed that the sport promotes the academic self-concept and it improves the academic achievement. On the other hand, the family self-concept is clearly affected when the subject use too much time to do physical activities. However, the hours devoted to sport do not directly influence academic achievement. Therefore, the importance of physical activity to improve self-concept and academic achievement is demonstrated.

Key words: self-concept; academic achievement; physical activity; basketball; school.

\section{Introducción}

Numerosas investigaciones establecen los beneficios que aporta la realización de actividad física sobre la salud, tanto de los componentes físicos, como sobre la mejora de aspectos psicológicos y sociales (Castro, Zurita, Martínez, Chacón y Espejo, 2016; Zurita, Castro-Sánchez, Álvaro, Rodríguez y Pérez, 2016), y es porque en algunos casos se afirma que las personas que realizan actividad física tienen un mayor rendimiento académico así como mejores valoraciones de las diferentes dimensiones del autoconcepto.

La práctica de actividad física, debe ser entendida como cualquier movimiento corporal que se produce por el conjunto de músculos del cuerpo y que conlleva un gasto energético, superior al estado de reposo, incluyéndose todo tipo de actividades que pueden ser realizadas en el entorno laboral, escolar o deportivo(Márquez, 1995; Luengo, 2007; Martínez et al., 2012).

La actividad física, se asocia a la obtención de dos tipos de

Dirección para correspondencia [Correspodence address]: Fátima Chacón Borrego. E-mail: fchacon@us.es beneficios y mejoras, por un lado tenemos los de índolepsicológicos, relacionado conel bienestar que el individuo siente antes y después de dicha práctica y con las posibles mejoras de aspectos psicológicos como el rendimiento académico, la confianza, la estabilidad emocional, el funcionamiento intelectual, estados de ánimo, autocontrol, sociabilidad, etc., (Márquez, 1995; Jiménez, Martínez, Miró y Sánchez, 2008), y también se producen beneficios físicos de salud, asociados y vinculados con la posible reducción y prevención de enfermedades cardiovasculares; disminuye el riesgo de sufrir fracturas debido a que reduce la pérdida de mineral óseo; fortalece los músculos que intervienen en la actividad física, mejorando el equilibrio, coordinación y agilidad, entre otros aspectos (Aparicio, Carbonell-Baeza y Delgado, 2010).

Además, la práctica regular de actividad física evita el ocio sedentario disminuyendo la posibilidad de padecer sobrepeso y otras enfermedades (Ara, Vicente-Rodríguez, Moreno, Gutin y Casajus, 2009; Moya et al., 2011; Amigo, Busto, Herrero y Fernández, 2008). 
Otro término a considerar en esta población según lo reportado por Musitu, García y Gutiérrez (1991) es el autoconcepto que puede ser entendido como la percepción que el individuo tiene de sí mismo, basado en sus experiencias con los que le rodean y en las propias atribuciones de su conducta. (González-Pienda, Núñez, González-Pumariega y García, 1997; Zurita et al., 2016). A su vezFlores, Medrano y Manoiloff (2014) especifican que el autoconcepto se conforma a partir de las creencias, hipótesis y supuestos que un individuo posee sobre sí mismo, los cuales, aportan estructura, coherencia y significado a la existencia personal. Este se apoya en la teoría multidimensional de Shavelson, Hubner y Stanton (1976), siendo la más aceptada a día de hoy en el ámbito de la investigación. De esta manera se puede medir el de tipo académico, referido a la percepción que la persona tiene sobre la importancia del rol que desempeńa como estudiante; el social, que mide la percepción que un individuo tiene de sus círculos y relaciones sociales; el emocional, condicionado por la percepción que el individuo tiene sobre su propio estado emocional y de sus posibles respuestas a situaciones concretas; el familiar, percepción que un individuo tiene sobre su implicación, participación e integración en el entorno familiar ypor último el físico, esta dimensión se refiere a la percepción que una persona tiene sobre su aspecto y nivel de condición física (García y Musitu, 1999).

Elrendimiento académico, se puede entender como el nivel de conocimientos que se han demostrado sobre una materia o área en una edad y nivel académico determinado de acuerdo a lo expuesto por García Jiménez, Alvarado y Jiménez (2000). Las diferentes investigaciones sobre este aspecto establecen la necesidad de tener en cuenta multitud de factores entre los que se encuentra, la acción individual del estudiante, la influencia del grupo/clase sobre el individuo, su contexto educativo, calificaciones escolares, inteligencias múltiples y aprendizajes escolares entre otros. Todos estos factores se pueden agrupar en tres dimensiones: motivación escolar, autocontrol del sujeto y habilidades sociales (Núñez et al., 1998; Castejón y Pérez, 1998; Edel, 2003). A este respecto Goñi, Ruiz de Azúa y Rodríguez (2004), comprueban una importante relación entre actividad física y autoconcepto, y consideran esencial el de tipo físico.

En el ámbito de la educación física se ha popularizado la evidencia de que la práctica deportiva favorece el desarrollo personal y, de igual manera, la idea de que entre autoconcepto y rendimiento deportivo se producen fuertes vinculaciones (Candel, Olmedilla yBlas, 2008; Guillén y Ramírez, 2010). Además la práctica de actividad físico-deportiva puede mejorar la participación del sujeto en sus círculos sociales fortaleciendo lazos afectivos y favorecer la evasión de problemas cotidianos. Por lo tanto, de acuerdo a la literatura científica, la actividad física continuada no sólo mejoraría el autoconcepto físico, sino que también, puede mejorar el autoconcep- to social y emociona. Igualmente la actividad físicafavorece la mejora del autoconcepto académico al conseguir un mejor funcionamiento del cerebro, con mejores estados de concentración y beneficia, por tanto, los procesos de aprendizaje según los trabajos de González y Tourón (1992); GonzálezPienda et al.(1997) o Carmona, Sánchez y Bakieva (2011).

Las diferentes investigaciones sobre actividad física y rendimiento académico muestran que la realización de actividades deportivas extraescolares se encuentra relacionada con la mejora de resultados en materias específicas como matemáticas e inclusive sobre la calificación final del curso académico (Cladellas, Clariana, Badia, Gotzens, 2015). En este mismo sentido Ramírez, Vinaccia y Suárez (2004) obtienen una mejora del rendimiento académico en aquellos sujetos que realizan una práctica continuada de actividad físico-deportiva a diferencia de personas más sedentarias, porque la actividad física favorece el funcionamiento cerebral y cognoscitivo. A este respecto la influencia del autoconcepto sobre el rendimiento académico puede llegar a ser inmediata, mientras que, la incidencia del logro escolar está mediatizada por la elaboración cognitivo-afectiva del propio autoconcepto (GonzálezPienda et al.,1997).

En este sentido el presente trabajo se centra en describir y analizar las variables psicológicas y dictaminar las posibles relaciones entre la práctica de actividad físico-deportiva extraescolar en jugadoras de baloncesto de categoría cadete, su autoconcepto y nivel de rendimiento académico.

\section{Método}

\section{Diseño y participantes}

Estudio de tipo descriptivo y relacional, de corte transversal. Está realizado sobre una muestra de 75 jugadoras de entre 12 y 16 años de edad, pertenecientes a 8 equipos de baloncesto de distintas localidades de España. La distribución de la muestra (número de jugadoras por equipo) fue la siguiente:: AeseHospitalet (8), Agustinos Eras León (9), Bosco Ourense (15), Miralvalle Plasencia (12), Presentación Granada (6), Canoe Madrid (11), San Blas Alicante (10) y Selección Cadete Granada (4), utilizando para ello una técnica de muestreo aleatorio incidental no probabilístico.

\section{Instrumentos}

Este trabajo utiliza el cuestionario como herramienta para la recogida de datos.

- Un cuestionario de autorregistrosociodemográfico, de práctica deportiva y datos de rendimiento académico que incluye ítems para las variables: edad de cada participante; club al que pertenece; horas de entrenamiento a la semana (h/sem.) (variable numérica codificada con 
los siguientes valores: 1 = hasta $3 \mathrm{~h} / \mathrm{sem}$; 2 = entre 3-6 h/sem.; 3 = entre 6-9 h/sem.; 4 = más de $9 \mathrm{~h} / \mathrm{sem}$.); la nota académicamedia de la última evaluación sobre una escala 1-10 (codificada en $0=$ Suspenso (valores entre 0 y 4), 1= Suficiente (valor 5), 2 = Bien (valor 6), 3 = Notable (7-8), 4 = Sobresaliente (9-10).

- El cuestionario AF-5 sobre el autoconcepto y sus distintas dimensiones (García y Musitu, 1999): Autoconcepto Académico (AA), Autoconcepto Social (AS), Autoconcepto Emocional (AE), Autoconcepto Familiar (AF) y Autoconcepto Físico (AFS). Contiene 6 dimensiones, con un total de 30 ítems con estructura pentadimensional $(1=$ nunca; $2=$ pocas veces; $3=$ algunas veces; $4=$ muchas veces; $5=$ =siempre). El coeficiente de consistencia interno alpha de Cronbachobtenido por los autores fue de $\alpha=, 840$, muy similar al obtenido en nuestro estudio $(\alpha=, 867)$.

\section{Procedimiento}

Se solicitó la participación de todas las jugadoras participantes en el torneo de baloncesto (133 sujetos), a las que se les entregó los cuestionarios y documento de consentimiento informado para participar en la investigación. Previamente se informa sobre la finalidad de la investigación y el carácter voluntario y anónimo de la misma.

Para la cumplimentación de los cuestionarios se indica que preferentemente lo hicieran a la finalización de la jornada deportiva y nunca en el transcurso de un partido ni en los tiempos inmediatos previos o posteriores al mismo. El número de cuestionarios válidos fueron 75 .

\section{Análisis de datos}

El tratamiento estadístico de los datos se realizó con el software SPSS en su versión 22.0 para Windows. Para realizar los análisis descriptivos de rendimiento académico y la actividad física se utilizaron frecuencias, porcentajes y medias. Y para las relaciones entre variables de tipo nominal y de escala se realizó la prueba de ANOVA de un factor, pues todos los factores disponían de más de dos categorías, además de una tabla de contingencias.

\section{Resultados}

El rendimiento académico de las jugadoras, teniendo en cuenta la calificación media obtenida en la última evaluación (Tabla 1), es que el $92 \%$ de la muestra tienen una media $\geq$ a 7 (58,7\% de notable y $33.3 \%$ de sobresaliente), siendo la calificación de suficiente y aprobado la obtenida por un porcentaje menor de representación (el 4\% respectivamente). Asimismo todas las jugadoras realizan una práctica deportiva extraesco- lar que requiere un compromiso en horas de entrenamiento semanal que oscila entre menos de 3 horas semanales y más de 9 (h/sem.). La mayoría de la muestra (el 57,3\%) entrena entre 6 y 9 h/sem., seguido de entre 3 y 6 h/sem. (26,7\%). Un 13,3\% lo hace más de $9 \mathrm{~h} / \mathrm{sem}$. y tan sólo el 2,7\% entrena menos de $3 \mathrm{~h} / \mathrm{sem}$.

Tabla 1. Datos descriptivos.

\begin{tabular}{cccc}
\hline Calificación & Porcentaje & $\begin{array}{c}\text { Horas entrenamiento/ } \\
\text { semana }\end{array}$ & Porcentaje \\
\hline Suspenso & - & Hasta 3 & $2,7 \%$ \\
Suficiente & $4,0 \%$ & Entre 3-6 & $26,7 \%$ \\
Bien & $4,0 \%$ & Entre 6-9 & $57,3 \%$ \\
Notable & $58,7 \%$ & Más de 9 & $13,3 \%$ \\
Sobresaliente & $33,3 \%$ & & \\
\hline
\end{tabular}

En la siguiente tabla se muestran los resultados descriptivos relativos a las diferentes dimensiones del autoconcepto. Encontramos mayores niveles de Autoconcepto Familiar (AF) con una media de 4,47, seguido del Autoconcepto Social (AS) con una $M=4,08$, y el Autoconcepto Académico (AA) $M=$ 3,90; los niveles más bajos de autoconcepto los obtenemos en la dimensión Emocional (AE) $M=3,47$ y Físico (AF) $M=$ 3,70 .

Tabla 2. Niveles de Autoconcepto General y Dimensiones.

\begin{tabular}{lcccc}
\hline & Mínimo & Máximo & Media & DT \\
\hline $\begin{array}{c}\text { Autoconcepto } \\
\text { General }\end{array}$ & 2,77 & 4,93 & 3,93 & 0,45 \\
$\begin{array}{c}\text { Autoconcepto } \\
\text { Académico }\end{array}$ & 1,50 & 5,00 & 3,90 & 0,68 \\
$\begin{array}{c}\text { Autoconcepto } \\
\quad \text { Social }\end{array}$ & 2,33 & 5,00 & 4,08 & 0,62 \\
$\begin{array}{c}\text { Autoconcepto } \\
\text { Emocional }\end{array}$ & 1,50 & 5,00 & 3,47 & 0,78 \\
$\begin{array}{c}\text { Autoconcepto } \\
\text { Familiar }\end{array}$ & 2,50 & 5,00 & 4,47 & 0,68 \\
$\begin{array}{c}\text { Autoconcepto } \\
\text { Físico }\end{array}$ & 2,17 & 5,00 & 3,70 & 0,55 \\
\hline
\end{tabular}

Nota: Niveles de autoconcepto medidos en una escala 0 a 5

La relación autoconcepto-rendimiento académico, dependiendo de la nota media (Tabla 3), determinaron diferencias estadísticamente significativas $(p \leq 0,05)$ en el Autoconcepto Académico $\left(p=0.000^{*}\right)$. El resto de categorías del autoconcepto no arrojan datos significativos, aunque atendiendo a las notas medias en la calificación podemos observar que los que tienen Sobresaliente tienen un mejor autoconcepto académico $(M=4,26)$; en el Autoconcepto Social hay valoraciones muy similares entre los que obtienen Suficiente, Bien, Notable o Sobresaliente $(M=4,05 ; M=4,11 ; M=4,08 ; M=$ 
4,09 respectivamente); al igual que ocurreen el Autoconcepto Emocional ( $M=3,77 ; M=3,38 ; M=3,37 ; M=3,63)$. Existe un menor nivel de Autoconcepto Familiar, en las jugadoras con una calificación de Suficiente $(\mathrm{M}=3,83)$. El Autoconcepto Físico es muy similar en todas las jugadoras independientemente de su calificación académica, aunque se observa que es un poco mejor en el grupo con una calificación de Bien $(\mathrm{M}=4,16)$.

Tabla 3. Niveles de Autoconcepto según nota media.

\begin{tabular}{|c|c|c|c|c|c|c|}
\hline & Nota Media & $\mathrm{N}$ & Media & DT & $\mathrm{F}$ & Sig. \\
\hline \multirow{4}{*}{$\begin{array}{c}\text { Auto- } \\
\text { concepto } \\
\text { Académico }\end{array}$} & Suficiente & 3 & 2,27 & 0,69 & \multirow{4}{*}{12,578} & \multirow{4}{*}{$0,000^{*}$} \\
\hline & Bien & 3 & 3,38 & 0,09 & & \\
\hline & Notable & 44 & 3,85 & 0,62 & & \\
\hline & Sobresaliente & 25 & 4,26 & 0,43 & & \\
\hline \multirow{4}{*}{$\begin{array}{c}\text { Auto- } \\
\text { concepto } \\
\text { Social }\end{array}$} & Suficiente & 3 & 4,05 & 0,25 & \multirow{4}{*}{0,005} & \multirow{4}{*}{0,999} \\
\hline & Bien & 3 & 4,11 & 0,78 & & \\
\hline & Notable & 44 & 4,08 & 0,66 & & \\
\hline & Sobresaliente & 25 & 4,09 & 0,60 & & \\
\hline \multirow{4}{*}{$\begin{array}{c}\text { Auto- } \\
\text { concepto } \\
\text { Emocional }\end{array}$} & Suficiente & 3 & 3,77 & 0,83 & \multirow{4}{*}{0,749} & \multirow{4}{*}{0,527} \\
\hline & Bien & 3 & 3,38 & 1,33 & & \\
\hline & Notable & 44 & 3,37 & 0,75 & & \\
\hline & Sobresaliente & 25 & 3,63 & 0,78 & & \\
\hline \multirow{4}{*}{$\begin{array}{c}\text { Auto- } \\
\text { concepto } \\
\text { Familiar }\end{array}$} & Suficiente & 3 & 3,83 & 1,09 & \multirow{4}{*}{0,946} & \multirow{4}{*}{0,423} \\
\hline & Bien & 3 & 4,38 & 1,05 & & \\
\hline & Notable & 44 & 4,50 & 0,60 & & \\
\hline & Sobresaliente & 25 & 4,50 & 0,73 & & \\
\hline \multirow{4}{*}{$\begin{array}{l}\text { Auto- } \\
\text { concepto } \\
\text { Físico }\end{array}$} & Suficiente & 3 & 3,83 & 0,92 & \multirow{4}{*}{1,393} & \multirow{4}{*}{0,252} \\
\hline & Bien & 3 & 4,16 & 0,16 & & \\
\hline & Notable & 44 & 3,61 & 0,52 & & \\
\hline & Sobresaliente & 25 & 3,80 & 0,56 & & \\
\hline
\end{tabular}

Al estudiar la relación entre los niveles de autoconcepto y la dedicación a la práctica de actividad físico-deportiva (PAFYD) medida en número de horas de entrenamiento a la semana (h/sem.), comprobamos que existe una relación significativa entre el Autoconcepto Familiar y el número de h/sem., de forma que hay un menor nivel en esta dimensión (AF) en las jugadoras que entrenan más de nueve horas $(M=3,83)$; en el resto de dedicaciones de PAFYD los niveles de AF están en una media entre 4,33 y 4,63 (para los que entrenan menos de $3 \mathrm{~h} / \mathrm{sem}$. y entre 6 y $9 \mathrm{~h} / \mathrm{sem}$. respectivamente).
Tabla 4. Niveles de Autoconcepto según Horas de Entrenamiento.

\begin{tabular}{|c|c|c|c|c|c|c|}
\hline & Horas/semana & $\mathrm{N}$ & Media & DT & $\mathrm{F}$ & Sig \\
\hline \multirow{4}{*}{$\begin{array}{c}\text { Auto- } \\
\text { concepto } \\
\text { Académico }\end{array}$} & Hasta 3 & 2 & 4,00 & 0,47 & \multirow{4}{*}{0,813} & \multirow{4}{*}{0,491} \\
\hline & Entre 3-6 & 20 & 3,79 & 0,68 & & \\
\hline & Entre 6-9 & 43 & 4,00 & 0,68 & & \\
\hline & Más de 9 & 10 & 3,70 & 0,71 & & \\
\hline \multirow{4}{*}{$\begin{array}{l}\text { Auto- } \\
\text { concepto } \\
\text { Social }\end{array}$} & Hasta 3 & 2 & 3,66 & 0,94 & \multirow{4}{*}{0,475} & \multirow{4}{*}{0,700} \\
\hline & Entre 3-6 & 20 & 4,10 & 0,65 & & \\
\hline & Entre 6-9 & 43 & 4,12 & 0,58 & & \\
\hline & Más de 9 & 10 & 3,96 & 0,77 & & \\
\hline \multirow{4}{*}{$\begin{array}{c}\text { Auto- } \\
\text { concepto } \\
\text { Emocional }\end{array}$} & Hasta 3 & 2 & 2,75 & 1,53 & \multirow{4}{*}{1,198} & \multirow{4}{*}{0,317} \\
\hline & Entre 3-6 & 20 & 3,37 & 0,74 & & \\
\hline & Entre 6-9 & 43 & 3,59 & 0,77 & & \\
\hline & Más de 9 & 10 & 3,30 & 0,78 & & \\
\hline \multirow{4}{*}{$\begin{array}{l}\text { Auto- } \\
\text { concepto } \\
\text { Familiar }\end{array}$} & Hasta 3 & 2 & 4,33 & 0,23 & \multirow{4}{*}{4,200} & \multirow{4}{*}{$0,009^{*}$} \\
\hline & Entre 3-6 & 20 & 4,47 & 0,67 & & \\
\hline & Entre 6-9 & 43 & 4,63 & 0,53 & & \\
\hline & Más de 9 & 10 & 3,83 & 0,97 & & \\
\hline \multirow{4}{*}{$\begin{array}{l}\text { Autocon- } \\
\text { cepto Físico }\end{array}$} & Hasta 3 & 2 & 3,16 & 0,00 & \multirow{4}{*}{0,757} & \multirow{4}{*}{0,522} \\
\hline & Entre 3-6 & 20 & 3,74 & 0,58 & & \\
\hline & Entre 6-9 & 43 & 3,69 & 0,57 & & \\
\hline & Más de 9 & 10 & 3,80 & 0,40 & & \\
\hline
\end{tabular}

En la última tabla se relacionan las variables horas de entrenamiento y calificación. Según los resultados obtenidos, aunque no son significativos, la cantidad de horas de actividad física más adecuada para obtener mejores calificaciones es de entre 6-9 h/sem., siendo la menos óptima la franja más baja de hasta $3 \mathrm{~h} / \mathrm{sem}$.

Tabla 6. Relación entre horas de entrenamiento semanal y calificación.

\begin{tabular}{cccccc}
\hline Calificación & \multicolumn{5}{c}{ Horas de entrenamiento } \\
\hline \multirow{3}{*}{ Suficiente } & & $\begin{array}{c}\text { Hasta } \\
\text { 3 horas }\end{array}$ & $\begin{array}{c}\text { Entre } \\
3-6 .\end{array}$ & $\begin{array}{c}\text { Entre } \\
\text { 6-9. }\end{array}$ & $\begin{array}{c}\text { Más } \\
\text { de 9 }\end{array}$ \\
\cline { 2 - 6 } & Recuento & 0 & 0 & 2 & 1 \\
\hline \multirow{2}{*}{ Bien del total } & $0,0 \%$ & $0,0 \%$ & $2,7 \%$ & $1,3 \%$ \\
\hline \multirow{3}{*}{ Notable } & Recuento & 0 & 2 & 0 & 1 \\
\cline { 2 - 6 } & \% del total & $0,0 \%$ & $2,7 \%$ & $0,0 \%$ & $1,3 \%$ \\
\cline { 2 - 6 } & Recuento & 1 & 16 & 22 & 5 \\
\hline \multirow{2}{*}{ Sobresaliente dotal } & $1,3 \%$ & $21,3 \%$ & $29,3 \%$ & $6,7 \%$ \\
\cline { 2 - 6 } & Recuento & 1 & 2 & 19 & 3 \\
\cline { 2 - 6 } & $\%$ del total & $1,3 \%$ & $2,7 \%$ & $25,3 \%$ & $4,0 \%$ \\
\hline
\end{tabular}




\section{Discusión y conclusiones}

Los resultados sobre autoconcepto de jugadoras cadete de baloncesto extraescolar reflejan como la dimensión autoconcepto familiar obtiene diferencias con respecto a las demás, estos resultados son similares a los estudios de Moreno y Cervelló (2005); Candel et al. (2008); Moreno (2008), en el resto de dimensiones las diferencias no son muy notables, como ocurre en el trabajo de Guillén y Ramírez (2010).

Con respecto a la relación entre autoconcepto y las calificaciones académicas, los resultados indican que son significativas en el autoconcepto académico, mostrando así que la práctica de la actividad física, mejora esta dimensión. Estos resultados coinciden con el estudio realizado por Cladellas et al. (2015) y Castro-López, Zagalaz Sánchez, Pérez-Gómez, y Cachón (2016), que revelan que los estudiantes que realizan actividades extraescolares de tipo recreativo obtienen mejores notas que aquellos que realizan actividades de tipo cognitivo; al igual que acontecía en los trabajos de Carmona et al. (2011), Ramírez et al. (2004) y González y Portolés (2014). Otros trabajos que avalan nuestros resultados son los de González-Pienda et al. (2002); Peralta y Sánchez (2003); Gallardo, Garfella, Sánchez, Ros y Carbonell (2009).

Los resultados sobre si existe una relación entre el autoconcepto y el número de horas dedicadas a la actividad física indican que son significativas en el autoconcepto familiar, de forma que los niveles de este autoconcepto se mantienen estables siempre y cuando no se supere una determinada cantidad de tiempo de actividad física a la semana (en nuestro caso siempre que no se entrene más de 9 horas semanales) a partir de la cual se reduce el nivel de esta dimensión. Estos resultados van en la misma línea que los obtenidos por Candel et al. (2008), (aunque en su trabajo la cantidad de actividad fue medida en función del número de días al mes) en los que obtienen que las chicas que practican deporte ocho días o menos al mes en comparación con las que lo realizan nueve días o más, obtienen mejores puntuaciones en el autoconcepto familiar y emocional propiciado por factores de imagen $y$ autopercepción de sí mismo (Contreras, Gil-Madrona, García, Fernández y Pastor, 2010; López Sánchez, López Sánchez y Díaz-Suárez, 2015, 2016). Debemos señalar que no existen evidencias científicas suficientes sobre la relación entre autoconcepto familiar y práctica deportiva con los que contrastar nuestros resultados.

Las principales conclusiones de este trabajo muestran que:

- Las jugadoras de baloncesto estudiadas tienen niveles de autoconcepto similares, tanto en el autoconcepto general como en las demás dimensiones, sólo destaca ligeramente el nivel de Autoconcepto Familiar.

- Los mayores porcentajes de la muestra obtienen calificaciones altas de notable y sobresaliente. Ninguna de las participantes en el estudio obtiene un suspenso en su nota media, ni ha repetido curso e igualmente hay porcentajes bajos en las calificaciones de suficiente y bien.

- La cantidad de horas de entrenamiento a la semana que se le dedica a este tipo de práctica deportiva extraescolar (no federada a excepción de las 4 jugadoras de la selección provincial) que más se utiliza es entre 6 y 9 horas, siendo la menos frecuente la inferior a 3 horas seguida de la de más de 9 horas.

- El autoconcepto general y en sus diferentes dimensiones es bueno, destaca sobre todas el autoconcepto familiar y social. La dimensión emocional, aunque con valores positivos, es la que tiene resultados más bajos.

- Sólo encontramos relaciones significativas entre la dimensión autoconcepto académico (AA) y las calificaciones de forma que es más alto a medida que las calificaciones son mejores.

- Sólo el Autoconcepto Familiar (AF) se ve directamente afectado por la cantidad de horas dedicadas a la práctica deportiva semanalmente, de forma que, sólo cuando hay un tiempo superior a 9 horas a la semana el AFse disminuye este autoconcepto.

- No es significativa la relación entre horas de entrenamiento y rendimiento académico.

\section{Referencias bibliográficas}

1. Amigo, I., Busto, R., Herrero, J. y Fernández, C. (2008). Actividad física, ocio sedentario, falta de sueño y sobrepeso infantil. Psicothema, 20(4), 516-520.

2. Aparicio, V. A., Carbonell-Baeza, A., y Delgado, M. (2010). Beneficios de la actividad física en personas mayores. Revista Internacional de Medicina y Ciencias de la Actividad Física y el Deporte, 10(40), 556-576.

3. Ara, I., Vicente-Rodríguez, G., Moreno, L. A., Gutin, B., yCasajus, J. A. (2009). La obesidad infantil se puede reducir mejor mediante actividad física vigorosa que mediante restricción calórica. Apunts. Medicina de l'Esport, 44(163), 111-118. http://doi.org/10.1016/S18866581(09)70118-5

4. Candel, N., Olmedilla, A. y Blas, A. (2008). Relaciones entre la práctica de actividad física y el autoconcepto, la ansiedad y la depresión en chicas adolescentes. Cuadernos de Psicología del Deporte, 8(1), 61-78.

5. Carmona, C., Sánchez, P., y Bakieva, M. (2011). Actividades extraescolares y rendimiento académico: diferencias en autoconcepto y género. Revista de Investigación Educativa, 29(2), 447-465.

6. Castejón, J.L., y Pérez, A.M. (1998). Un modelo casual-explicativo sobre la influencia de las variables psicosociales en el rendimiento académico. Bordón. Revista de pedagogía, 50(2), 171-185.

7. Castro, M., Zurita, F., Martínez, A., Chacón, R., y Espejo, T. (2016). Clima motivacional de los adolescentes y su relación con el género, la práctica de actividad física, la modalidad deportiva, la práctica deportiva federada y la actividad física familiar. Revista Internacional de Ciencias del Deporte, 12(45), 262-277. 
8. Castro-López, R., Zagalaz Sánchez, M. L., Pérez Gómez, V., y Cachón Zagalaz, J. (2016). Valoración de la relación entre Rendimiento Académico y Condición Física en escolares zaragozanos. SPORT TK-Revista EuroAmericana de Ciencias del Deporte, 5(1), 47-53.

9. Cladellas, R., Clariana, M., Badia, M., y Gotzens, C. (2015). Actividades extraescolares y rendimiento académico en alumnos de primaria. European Journal of Investigation in Health, Psychology and Education, 3(2), 87-97.

10. Contreras, O.R., Gil-Madrona, P., García, L.M., Fernández, J.G., y Pastor, J.C. (2010). Incidencia de un programa de Educación Física en la percepción de la propia imagen corporal. Revista de Educación, 357, 281-303.

11. Edel, R. (2003). El rendimiento académico: concepto, investigación y desarrollo. Revista Electrónica Iberoamericana sobre Calidad, Eficacia y Cambio en Educación 1(2), 1-15.

12. Flores, P. E., Medrano, L. A., y Manoiloff, L. M. (2014). Estados de ánimo y juicios de autoconcepto en universitarios: análisis desde un abordaje basado en redes semánticas naturales. Revista Interamericana de Psicología, 48(2), 291-307.

13. Gallardo, B., Garfella, P., Sánchez, F., Ros, C. y Carbonell, B. S. (2009). La influencia del autoconcepto en el rendimiento académico en estudiantes universitarios. Revista Española de Orientación y Psicopedagogía, 20(1), 16-28.

14. Garaigordobil, M. y Durá, A. (2006). Relaciones de autoconcepto y la autoestima con sociabilidad, estabilidad emocional y responsabilidad en adolescentes de 14 a 17 años. Análisis y Modificación de Conducta, 32(141).

15. García Jiménez, M. V., Alvarado, J. M. y Jiménez, A. (2000). La predicción del rendimiento académico: regresión lineal versus regresión logística. Psicothema, 12(2), 248-252.

16. García, F. y Musitu, G. (1999). AF5. Autoconcepto forma 5. Madrid: TEA ediciones.

17. González, J., y Portolés, A. (2014). Actividad física extraescolar: relaciones con la motivación educativa, rendimiento académico y conductas asociadas a la salud. Revista Iberoamericana de Psicología del Ejercicio y el Deporte, 9 (1), 51-65.

18. González, M. C. y Tourón, J. (1992). Autoconcepto y rendimiento escolar: sus implicaciones en la motivación y en la autorregulación del aprendizaje. Pamplona: Eunsa.

19. González-Pienda, J. A., Núñez, J. C., González-Pumariega, S., y García, M. (1997). Autoconcepto, autoestima y aprendizaje escolar. Psicothema, 9(2), 271-289.

20. González-Pienda, J. A., Núńez, J. C., Álvarez, L., González-Pumariega S., Roces, C., González, P., Bernardo, A. (2002). Inducción parental a la autorregulación, autoconcepto y rendimiento académico. Psicothema, $14(4), 853-860$

21. Gońi, A., Ruiz de Azúa, S.y Rodríguez, A. (2004). Deporte y autoconcepto físico en la preadolescencia. Apunts. Educación física y deportes, 3(77), 18-24.

22. Guillen, F., y Ramírez, M. (2010). Relación entre Autoconcepto y Condición Física en Alumnos del Tercer Ciclo de Primaria. Revista de Psico- logia del Deporte, 20(1), 45-59.

23. Jiménez, M. G., Martínez, P., Miró, E. y Sánchez, A. I. (2008). Bienestar psicológico y hábitos saludables: ¿están asociados a la práctica de ejercicio físico? International Journal of Clinical and Health Psychology, 8(1), 185-202.

24. López Sánchez, L., López Sánchez, G. F. y Díaz Suárez, A. (2015) Effects of a physical activity program on the body image of schoolchildren with ADHD. Cuadernos de Psicología del Deporte, 15(2), 135-142.

25. López Sánchez, G. F., López Sánchez, L. \& Díaz Suárez, A. (2016). Effects of a physical activity program on the general dynamic and segmentary coordination of children with ADHD. Journal of Sport and Health Research, 8(2), 115-128.

26. Luengo, C. (2007). Actividad físico-deportiva extraescolar en alumnos de primaria. Revista Internacional de Medicina y Ciencias de la Actividad Física y del Deporte, 7(27), 174-184.

27. Márquez, S. (1995). Beneficios psicológicos de la actividad física. Revista de psicología general y aplicada: Revista de la Federación Española de Asociaciones de Psicología, 48(1), 185-206.

28. Martínez, A., Chillón, P., Martín-Matillas, M., Pérez, I., Castillo, R., Zapatera, B. y Delgado-Fernández, M. (2012). Motivos de abandono y no práctica de actividad físico-deportiva en adolescentes españoles: estudio Avena. Cuadernos de Psicología del Deporte, 12(1), 45-54.

29. Moreno, J. A. (2008). Importancia de la práctica físico-deportiva y del género en el autoconcepto físico de los 9 a los 23 años. Internacional Journal of Clinical and Health Psychology, 8(1), 171-183.

30. Moreno, J. A., y Cervelló, E. (2005). Physical self-perception in spanish adolescents: effects of gender and involvent in physical activity. Journal of Human Movement Studies, 48, 291-311

31. Moya, P., Sánchez López, M., López, J., Escribano, F., Notario, B., Sal cedo, F., y Martínez, V. (2011). Coste-efectividad de un programa de actividad física de tiempo libre para prevenir el sobrepeso y la obesidad en niños de 9-10 ańos. Gaceta Sanitaria, 25(3), 198-204.

32. Musitu, G., García, F. y Gutiérrez, M. (1991). Autoconcepto FormaA. Madrid: TEA.

33. Núñez, J. C., González-García, J. A., García-Rodríguez, M. S., González-Pumariega, S., Roces, C., Álvarez, L., y González-Torres, M. C. (1998). Estrategias de aprendizaje, autoconcepto y rendimiento académico. Psicothema, 10(1), 97-109.

34. Peralta, F. J. y Sánchez, M. D. (2003). Relaciones entre el autoconcepto y el rendimiento académico, en alumnos de Educación Primaria. Electronic journal of research in educational psychology, 1(1), 95-120.

35. Ramírez, W., Vinaccia, S., y Suárez, G. R. (2004). El impacto de la actividad física y el deporte sobre la salud, la cognición, la socialización y el rendimiento académico: una revisión teórica. Revista de estudios sociales, 18, 67-75.

36. Shavelson, J., Hubner, J. J., y Stanton, G. C. (1976). Self-concept: validation of construct interpretations. Review of Educational Research, 46, 407-442.

37. Zurita, F., Castro-Sánchez, M., Álvaro, I., Rodríguez, S., y Pérez, A. (2016). Autoconcepto, actividad física y familia: un modelo de ecuaciones estructurales. Revista de Psicología del Deporte, 25(1), 97-104. 